\title{
Every flock generalised quadrangle has a hemisystem
}

\author{
John Bamberg, Michael Giudici and Gordon F. Royle
}

\begin{abstract}
We prove that every flock generalised quadrangle contains a hemisystem, and we provide a construction method which unifies our results with the examples of Cossidente and Penttila in the classical case.
\end{abstract}

\section{Introduction}

In 1965, Segre 22 introduced the notion of a hemisystem, that is a set of lines which contains exactly half the lines on each point, in his work on regular systems of the Hermitian surface. Segre proved that there is just one hemisystem of lines (up to equivalence) of the classical generalised quadrangle $\mathrm{H}\left(3,3^{2}\right)$, and thirty years later, it was conjectured by J. A. Thas [24, pp. 333] that there are no hemisystems of $\mathrm{H}\left(3, q^{2}\right)$ for $q>3$. However, forty years after Segre's seminal paper, Cossidente and Penttila 12 constructed, for each odd prime power $q$, a hemisystem of $\mathrm{H}\left(3, q^{2}\right)$. Since this work, hemisystems have received renewed attention in finite geometry (see $\mathbf{2 5}, \S 4]$ ). The concept of a hemisystem was extended by Cameron, Delsarte and Goethals [10 to all generalised quadrangles with the same parameters $\left(s^{2}, s\right)$ as the Hermitian surface, and in addition, they showed that such an object gives rise to a partial quadrangle, and hence also, to a strongly regular graph.

The only known generalised quadrangles of order $\left(s^{2}, s\right), s$ odd, are the flock generalised quadrangles, and we know that for $s$ prime, these are the only elation generalised quadrangles with these parameters (see [6]). There are nine known infinite families of such generalised quadrangles, and at least forty other examples for $q \leqslant 61$ (see [7]). As far as the authors are aware, the known hemisystems of these generalised quadrangles are listed in [3, [5, 11 and [12. In particular, only one hemisystem of a nonclassical flock generalised quadrangle has previously been constructed (in $\mathbf{3}$ ). It is the main purpose of this work to establish the following:

Theorem 1.1. Every flock generalised quadrangle of order $\left(s^{2}, s\right), s$ odd, contains a hemisystem.

If $\mathcal{H}$ is a hemisystem of a generalised quadrangle of order $\left(s^{2}, s\right)$, then we can build a partial quadrangle by taking the point-set to be $\mathcal{H}$, and the line-set to be the points of the generalised quadrangle. The pointgraph of a partial quadrangle is strongly regular and the small number of constructions of such graphs are outlined later in this section. The complement of a hemisystem is again a hemisystem, however, the two strongly regular graphs arising may not necessarily be isomorphic (see $\mathbf{3}$ for an example of such an instance). A hemisystem also gives rise to a cometric 4-class association scheme that is not metric, which are indeed rare in the literature (see [18]).

We will explain precisely what the flock generalised quadrangles are in Section 2 but we stress here that we rely heavily on the geometric model of Knarr [15. This yields flock generalised quadrangles from substructures known as BLT-sets of the symplectic polar space $\mathrm{W}(5, q)^{1}$. The linear BLT-set yields the classical flock generalised quadrangle $\mathrm{H}\left(3, q^{2}\right)$ via this construction, and varying the BLT-set gives all flock generalised quadrangles. We interpret the Cossidente-Penttila hemisystems of $\mathrm{H}\left(3, q^{2}\right)$ in this model, and show that analogously, varying the BLT-set produces hemisystems of the resulting nonclassical flock generalised quadrangles. Our construction produces a considerable number of non-isomorphic hemisystems of flock generalised quadrangles, but computer work (reported in [4]) shows that not every hemisystem arises from this method.

2000 Mathematics Subject Classification. 05B25 (primary), 05E30, 51E12 (secondary).

${ }^{1}$ Strictly speaking, a BLT-set is a set of lines of $\mathrm{W}(3, q)$, but we can identify them with a set of planes incident with a point of $\mathrm{W}(5, q)$. 
As mentioned earlier, hemisystems produce partial quadrangles. Partial quadrangles were introduced by Peter J. Cameron [9] as a geometry of points and lines such that every two points are on at most one line, there are no triangles, every line has the same number $s+1$ of points, every point is incident with the same number $t+1$ of lines, and there is a constant $\mu$ such that for every pair of noncollinear points there are precisely $\mu$ points collinear to both. It follows directly from these conditions that the point graph of this geometry is strongly regular with parameters ${ }^{2}$

$$
(1+s(t+1)(\mu+s t) / \mu, s(t+1), s-1, \mu) .
$$

In fact, generalised quadrangles themselves are partial quadrangles with $\mu=t+1$, that is, they satisfy the extra condition that for every point $P$ and line $\ell$ which are not incident, there is a unique point of $\ell$ collinear with $P$. The only known partial quadrangles which are not generalised quadrangles are:

- one of the seven known triangle-free strongly regular graphs,

- one of three exceptional examples arising from caps of projective spaces,

- those which arise from removing a point and its incident lines from a generalised quadrangle of order $\left(s, s^{2}\right)$, or lastly,

- those which arise from a hemisystem of a generalised quadrangle of order $\left(s^{2}, s\right), s$ odd. A partial quadrangle arising from a hemisystem has parameters $\mathrm{PQ}\left((s-1) / 2, s^{2},(s-1)^{2} / 2\right)$.

Finally, in the Appendix, we provide a new proof that a hemisystem of a generalised quadrangle of order $\left(s^{2}, s\right), s$ odd, gives rise to a partial quadrangle. The method of [10 was to show that the adjacency matrix of the point graph had three distinct eigenvalues, whereas we adopt a more direct approach and make use of some basic algebraic combinatorics.

\section{BLT-sets and the Knarr model}

2.1. Polar spaces and generalised quadrangles. By a theorem of Jacques Tits, every finite polar space is either a generalised quadrangle or arises from a finite vector space equipped with a nondegenerate reflexive sesquilinear form or a quadratic form. By Witt's Theorem, the maximal totally isotropic subspaces have the same algebraic dimension, which is called the rank of the polar space. We will assume that the reader is familiar with the fundamental theory of polar spaces, and we refer the reader to 14 for more details. If we take the totally isotropic subspaces containing a given subspace of a finite polar space, we obtain the quotient polar space of the same type but lesser rank. We use projective notation for polar spaces so that they differ from the standard notation for their collineation groups. For example, the notation $\mathrm{W}(d-1, q)$ denotes the symplectic polar space derived from the vector space $\mathrm{GF}(q)^{d}$ equipped with a non-degenerate alternating form. Here is a summary of the notation we will use for polar spaces.

\begin{tabular}{ll|ll}
\hline Polar Space & Notation & Polar Space & Notation \\
\hline Symplectic & $\mathrm{W}(d-1, q), d$ even & Orthogonal, elliptic & $\mathrm{Q}^{-}(d-1, q), d$ even \\
Hermitian & $\mathrm{H}\left(d-1, q^{2}\right)$ & Orthogonal, parabolic & $\mathrm{Q}(d-1, q), d$ odd \\
& & Orthogonal, hyperbolic & $\mathrm{Q}^{+}(d-1, q), d$ even \\
\hline
\end{tabular}

Table 1: Notation for the finite polar spaces.

The set of zeros of a homogeneous quadratic equation $\sum a_{i j} X_{i} X_{j}=0$ in $n$ variables over $\mathrm{GF}(q)$ defines a quadric of the projective space $\mathrm{PG}(n-1, q)$, and we say that a subspace is singular with respect to the quadric if all of its points are in the quadric. The associated bilinear form for the quadratic form $Q$ defining the quadric is given by $B_{Q}(u, v)=Q(u+v)-Q(u)-Q(v)$. The radical of $B_{Q}$ is the set of vectors which are orthogonal to every other vector of the ambient vector space, and if 0 is the only zero of $Q$ in the radical of $B_{Q}$, then we say that the quadric is non-singular and singular otherwise. Non-singular quadrics come in three different types depending on the dimension and Witt index of the quadric. In $\mathrm{PG}(2 m, q)$, there is only one non-singular quadric up to equivalence (of the collineation group) and it is often denoted $\mathrm{Q}(2 m, q)$; the parabolic quadric of $\mathrm{PG}(2 m, q)$ with Witt index $m$. In odd dimension, we have the elliptic and hyperbolic quadrics $\mathrm{Q}^{-}(2 m+1, q)$ and $\mathrm{Q}^{+}(2 m+1, q)$ respectively, and they have Witt indices $m$ and $m+1$ (resp.).

\footnotetext{
${ }^{2}$ We use the standard notation for strongly regular graphs. The parameters $(v, k, \lambda, \mu)$ give us the number of vertices $v$, the valency $k$, and the constants $\lambda$ and $\mu$ are the number of vertices adjacent to a pair of adjacent or nonadjacent vertices, respectively.
} 
The (thick) classical generalised quadrangles are those which arise from equipping a vector space with an alternating, quadratic or Hermitian form, and they are $\mathrm{W}(3, q), \mathrm{Q}(4, q), \mathrm{H}\left(3, q^{2}\right), \mathrm{Q}^{-}(5, q)$ and $\mathrm{H}\left(4, q^{2}\right)$. By taking the totally singular points and lines of the ambient projective space, we obtain the points and lines for the given classical generalised quadrangle. For example, $\mathrm{W}(3, q)$ is the incidence structure of all points of $\mathrm{PG}(3, q)$ and totally isotropic lines with respect to a null polarity ${ }^{3}$ and is a generalised quadrangle of order $(q, q)$. The point-line dual of $\mathrm{W}(3, q)$ is $\mathrm{Q}(4, q)$, the parabolic quadric of PG $(4, q)$, and is therefore a generalised quadrangle of order $(q, q)$ (see [21, 3.2.1]).

2.2. Flocks of quadratic cones and BLT-sets. A quadratic cone of $\mathrm{PG}(3, q)$, $q$ odd, consists of the $q+1$ lines joining a point $v$ (the vertex) to the points of a conic of a hyperplane not on $v$, together with the points lying on these lines. A flock of the quadratic cone $\mathcal{C}$ with vertex $v$ in $\mathrm{PG}(3, q)$ is a partition of the points of $\mathcal{C} \backslash\{v\}$ into conics. J. A. Thas [23 showed that a flock gives rise to a generalised quadrangle of order $\left(q^{2}, q\right)$, which we call a flock generalised quadrangle. A BLT-set of lines of $\mathrm{W}(3, q)$ is a set $\mathcal{B}$ of $q+1$ lines of $\mathrm{W}(3, q)$ such that no line of $\mathrm{W}(3, q)$ is concurrent with more than two lines of $\mathcal{B}$. Note that a line of $\mathrm{W}(3, q)$ not in $\mathcal{B}$ must be concurrent with either 0 or 2 elements of $\mathcal{B}$, so BLT-sets are sometimes known as (0,2)-sets. In [2], it was shown that, for $q$ odd, a flock of a quadratic cone in $\mathrm{PG}(3, q)$ gives rise to a BLT-set of lines of $\mathrm{W}(3, q)$. Conversely, a BLT-set gives rise to possibly many flocks, however we only obtain one flock generalised quadrangle up to isomorphism (see [20]).

For $q$ odd, Knarr [15] gave a direct geometric construction of a flock generalised quadrangle from a BLT-set of lines of $\mathrm{W}(3, q)$. If we were to construct a flock generalised quadrangle (see below 2.3) from a linear BLT-set of lines of $\mathrm{W}(3, q)$ (i.e., the $q+1$ lines obtained from field reduction of a Baer subline), we would obtain a generalised quadrangle isomorphic to the classical object $\mathrm{H}\left(3, q^{2}\right)$. The BLT-sets of lines of $\mathrm{W}(3, q)$ have been classified by Law and Penttila [17. for prime powers $q$ at most 29, and this has recently been extended by Betten [7] to $q \leqslant 61$.

2.3. The Knarr model. We will require familiarity with the symplectic polar space $\mathrm{W}(5, q)$ of rank 3. This geometry simply arises from taking the one, two and three-dimensional vector subspaces of $\mathrm{GF}(q)^{6}$ for which a given alternating bilinear form restricts to the zero form (i.e., the totally isotropic subspaces). For example, one can take this alternating bilinear form to be defined by

$$
\beta(x, y)=x_{1} y_{6}-x_{6} y_{1}+x_{2} y_{5}-x_{5} y_{2}+x_{3} y_{4}-x_{4} y_{3}
$$

This bilinear form also gives us a null polarity $\perp$ of the ambient projective space $\mathrm{PG}(5, q)$, defined by $U \mapsto U^{\perp}:=\left\{v \in \operatorname{GF}(q)^{6} \mid \beta(u, v)=0\right.$ for all $\left.u \in U\right\}$. We will now spend some time revising the Knarr construction of a flock generalised quadrangle as it is the model that we use in this paper to analyse hemisystems of flock generalised quadrangles. The ingredients of the Knarr construction are as follows:

- a null polarity $\perp$ of $\mathrm{PG}(5, q)$;

- a point $P$ of $\mathrm{PG}(5, q)$;

- a BLT-set of lines $\mathcal{O}$ of $\mathrm{W}(3, q)$.

Note that the totally isotropic lines and planes incident with $P$ yield the quotient polar space $P^{\perp} / P$ isomorphic to $\mathrm{W}(3, q)$. So we will abuse notation and identify $\mathcal{O}$ with a set of totally isotropic planes on $P$. Then we construct a generalised quadrangle $\mathcal{K}(\mathcal{O})$ from the objects in the table below and incidence inherited from that of $\mathrm{PG}(5, q)$.

\begin{tabular}{|c|c|c|c|}
\hline & Points & & Lines \\
\hline (i) & points of $\mathrm{PG}(5, q)$ not in $P^{\perp}$ & & $\begin{array}{l}\text { totally isotropic planes not on } P \text { and } \\
\text { meeting some element of } \mathcal{O} \text { in a line }\end{array}$ \\
\hline $\begin{array}{l}\text { (ii) } \\
\text { (iii) }\end{array}$ & $\begin{array}{l}\text { lines not incident with } P \text { but con- } \\
\text { tained in some element of } \mathcal{O} \\
\text { the point } P\end{array}$ & (b) & elements of $\mathcal{O}$ \\
\hline
\end{tabular}

The point $P$ is the base point of the flock generalised quadrangle. It turns out (see Payne's article [19. Proposition 3], and in particular, [16. Chapter 5]), that if the flock generalised quadrangle $\mathcal{K}(\mathcal{O})$ is nonclassical, then its automorphism group is of the form $(q-1) \cdot\left(E_{q} \rtimes K\right)$ where $E_{q}$ is the Heisenberg group of order $q^{5}$ with a centre of order $q, K$ is the stabiliser of the BLT-set $\mathcal{O}$ within PГSp $(4, q)$ and the cyclic group of order $q-1$ on the bottom gives us the kernel of the action on the lines through the

\footnotetext{
${ }^{3} \mathrm{~A}$ polarity $\rho$ of a projective space is an involutory inclusion-reversing permutation. Furthermore, $\rho$ is a null polarity if every point $P$ of the projective space is incident with its image $P^{\rho}$.
} 
base point; actually there is an exception in the case of the Kantor-Knuth flock generalised quadrangles in which the kernel could be two or four times larger.

2.4. An equivalence relation on a BLT-set. In a generalised quadrangle, the trace of two noncollinear points $x$ and $y$ is the set of all points collinear to both $x$ and $y$ (see [21, pp. 2]), and it is often denoted by $\{x, y\}^{\perp}$ or $\operatorname{Tr}(x, y)$. Similarly, we can define the trace $\operatorname{Tr}(\ell, m)$ of two disjoint lines $\ell$ and $m$ as the set of all lines concurrent with both $\ell$ and $m$. So in $\mathrm{W}(3, q)$, the trace of any pair of disjoint lines consists of $q+1$ lines. The following lemma follows from $\S 1.3 .6$ and $\S 3.3 .1$ of 21 and will be fundamental in proving Lemma 2.4 .

Lemma 2.1. Let $\ell_{1}, \ell_{2}, \ell_{3}$ be three pairwise disjoint lines of $\mathrm{W}(3, q), q$ odd. Then

$$
\left|\operatorname{Tr}\left(\ell_{1}, \ell_{2}\right) \cap \operatorname{Tr}\left(\ell_{1}, \ell_{3}\right)\right| \in\{0,2\} .
$$

Given a line which is not concurrent with any member of a BLT-set of lines $\mathcal{B}$ of W $(3, q)$, we obtain an interesting equivalence relation on the elements of $\mathcal{B}$ (Definition 2.3), and in order to prove that we indeed have such an equivalence relation, we resort to working in the dual situation in the parabolic quadric $\mathrm{Q}(4, q)$. The notional advantages of working in this setting are the perceived "extra" geometric structures that we have at our disposal. This duality arises from the Klein correspondence between the lines of $\mathrm{PG}(3, q)$ and $\mathrm{Q}^{+}(5, q)$ (see $\mathbf{1 3}$ ). For example, the dual of a regulus of $\mathrm{W}(3, q)$ is a conic of $\mathrm{Q}(4, q)$, and in some sense, non-degenerate subspaces are easier to work with than sets of lines. There are two isometry types for non-degenerate lines with respect to a non-singular quadric $\mathcal{Q}$, namely, (i) lines containing no points of $\mathcal{Q}$ (external lines) and (ii) lines containing two points of $\mathcal{Q}$ (secant lines). The following lemma is needed for the proof of Lemma 2.4

Lemma 2.2. Let $\mathcal{Q}$ be a non-singular quadric of $\mathrm{PG}(4, q)$, $q$ odd, and let $e$ be a line of $\mathrm{PG}(4, q)$ external to $\mathcal{Q}$. Take three distinct points $b, b^{\prime}$ and $b^{\prime \prime}$ of the non-singular conic $e^{\perp} \cap \mathcal{Q}$ and define the following non-degenerate planes:

$$
\pi_{1}=\left\langle b, b^{\prime}\right\rangle^{\perp}, \quad \pi_{2}=\left\langle b^{\prime}, b^{\prime \prime}\right\rangle^{\perp}, \quad \pi_{3}=\left\langle b^{\prime \prime}, b\right\rangle^{\perp} .
$$

Let $C$ be a degenerate hyperplane meeting $\pi_{1}$ in a secant line. Then the non-degenerate lines $C \cap \pi_{2}$ and $C \cap \pi_{3}$ are of different isometry types.

Proof. Suppose $\mathcal{Q}$ is defined by the quadratic form

$$
Q(x)=x_{1} x_{5}+x_{2} x_{4}+x_{3}^{2} .
$$

Without loss of generality, we may chose $e$ to be the line $\langle(1,0,0,0, n),(0,0,1,0,0)\rangle$ where $n$ is some fixed non-square of $\operatorname{GF}(q)$, and since the stabiliser of a conic in $\operatorname{PO}(5, q)$ is 3-transitive on the conic, we may suppose that $b, b^{\prime}$ and $b^{\prime \prime}$ are the following points of $e^{\perp} \cap \mathcal{Q}$ :

$$
b=(0,1,0,0,0), \quad b^{\prime}=(0,0,0,1,0), \quad b^{\prime \prime}=(-1,1,0, n, n) .
$$

Therefore, we see that

$$
\pi_{1}=\langle(1,0,0,0,0), e\rangle, \quad \pi_{2}=\langle(-1,0,0, n, 0), e\rangle, \quad \pi_{3}=\langle(-1,1,0,0,0), e\rangle .
$$

Now a secant line of $\pi_{1}$ must have nonzero projections to two of the first, third and fifth coordinates. Let $v$ be a point of $\mathcal{Q}$ not in $\pi_{1}$. Below we will identify lines with $2 \times 5$ matrices, whereby the rows of the matrix give us the row-space of the associated 2-dimensional vector subspace. Let $M$ be the Gram matrix for the bilinear form arising from $Q$. Now $v^{\perp}$ meets $\pi_{1}$ in a line $s_{v}$ :

$$
s_{v}=v^{\perp} \cap \pi_{1}=\left[\begin{array}{ccccc}
-v_{1} & 0 & 0 & 0 & v_{5} \\
-2 v_{3} & 0 & v_{5} & 0 & 0
\end{array}\right]
$$

which is a secant if and only if $\operatorname{det}\left(s_{v} M s_{v}^{T}\right)$ is a square. We have

$$
\operatorname{det}\left(s_{v} M s_{v}^{T}\right)=\operatorname{det}\left[\begin{array}{cc}
-2 v_{1} v_{5} & -2 v_{3} v_{5} \\
-2 v_{3} v_{5} & 2 v_{5}^{2}
\end{array}\right]=-4 v_{1} v_{5}^{3}-4 v_{3}^{2} v_{5}^{2}=-4 v_{5}^{2}\left(v_{1} v_{5}+v_{3}^{2}\right)=4 v_{5}^{2}\left(v_{2} v_{4}\right) .
$$

and hence $v_{2} v_{4} \in \square$. Now

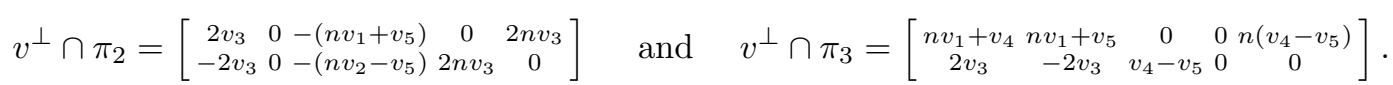

We want to show that $\operatorname{det}\left(\left(v^{\perp} \cap \pi_{2}\right) M\left(v^{\perp} \cap \pi_{2}\right)^{T}\right)$ and $\operatorname{det}\left(\left(v^{\perp} \cap \pi_{3}\right) M\left(v^{\perp} \cap \pi_{3}\right)^{T}\right)$ are not both square and not both non-square. These determinants turn out to be respectively:

$$
\begin{aligned}
& \Delta_{2}=16 n^{2} v_{3}^{2}\left(n v_{1} v_{2}+n v_{2}^{2}-v_{3}^{2}-v_{1} v_{5}-v_{2} v_{5}\right)=16 n^{2} v_{3}^{2} v_{2}\left(n\left(v_{1}+v_{2}\right)+v_{4}-v_{5}\right) \\
& \Delta_{3}=4 n\left(v_{4}-v_{5}\right)^{2}\left(-n\left(v_{1} v_{5}+v_{3}^{2}\right)+n v_{1} v_{4}+v_{4}^{2}-v_{4} v_{5}\right)=4 n\left(v_{4}-v_{5}\right)^{2} v_{4}\left(n\left(v_{1}+v_{2}\right)+v_{4}-v_{5}\right)
\end{aligned}
$$


So the product of $\Delta_{2}$ and $\Delta_{3}$ is

$$
8^{2} n^{3} v_{3}^{2}\left(v_{4}-v_{5}\right)^{2}\left(n\left(v_{1}+v_{2}\right)+v_{4}-v_{5}\right)^{2}\left(v_{2} v_{4}\right)
$$

and since $v_{2} v_{4} \in \square$, we see that $\Delta_{2} \Delta_{3}$ is simply a product of $n$ and a square. Therefore $\Delta_{2} \Delta_{3}$ is a non-square and hence the lines $v^{\perp} \cap \pi_{2}$ and $v^{\perp} \cap \pi_{3}$ are of different isometry types with respect to the quadric $\mathcal{Q}$; thus completing the proof.

Definition 2.3. Let $\mathcal{B}$ be a BLT-set of lines of $\mathrm{W}(3, q)$ and let $\ell$ be a line of $\mathrm{W}(3, q)$ disjoint from every member of $\mathcal{B}$. Define a binary relation $\equiv_{\ell}$ on $\mathcal{B}$ by setting $b \equiv_{\ell} b^{\prime}$ if and only if

$$
b=b^{\prime} \text { or } \operatorname{Tr}(b, \ell) \cap \operatorname{Tr}\left(b^{\prime}, \ell\right)=\varnothing .
$$

Lemma 2.4. The relation $\equiv_{\ell}$ given by Definition 2.3 is an equivalence relation with two equivalence classes of equal size.

Proof. First note that $\equiv_{\ell}$ is trivially reflexive and symmetric, so we will prove that $\equiv_{\ell}$ is transitive (by using the contrapositive statement of the standard definition). Suppose we have three elements $b, b^{\prime}, b^{\prime \prime}$ of $\mathcal{B}$ such that $\operatorname{Tr}(b, \ell) \cap \operatorname{Tr}\left(b^{\prime \prime}, \ell\right) \neq \varnothing$, that is, there is some line $m$ meeting $\ell, b$ and $b^{\prime \prime}$. To establish transitivity, we will show that $\operatorname{Tr}\left(b^{\prime}, \ell\right)$ must meet either $\operatorname{Tr}(b, \ell)$ or $\operatorname{Tr}\left(b^{\prime \prime}, \ell\right)$. Let us look at the dual situation in the parabolic quadric $\mathrm{Q}(4, q)$, and write $x^{D}$ for the dual object corresponding to $x$.
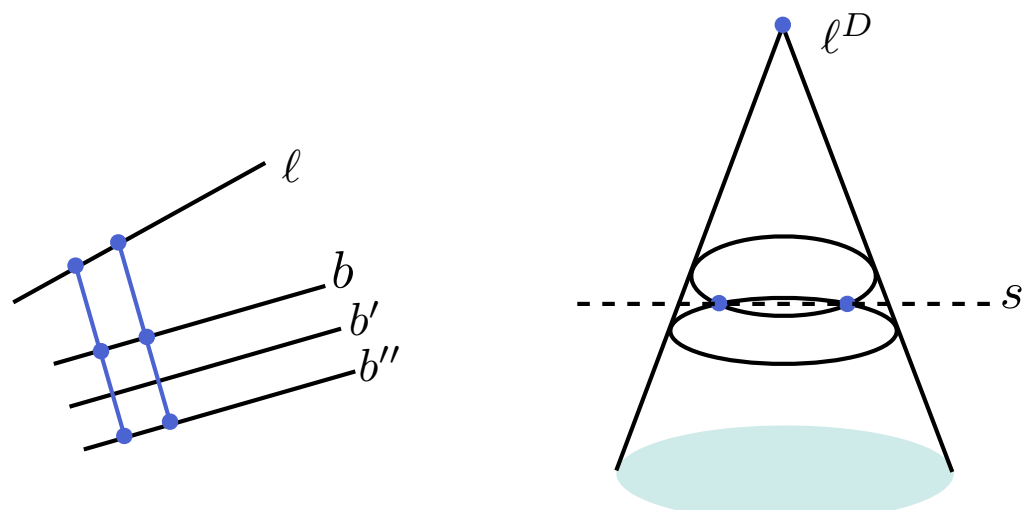

Figure 1: The dual situation.

Then $\ell$ corresponds to a point $\ell^{D}, \operatorname{Tr}(b, \ell)$ and $\operatorname{Tr}\left(b^{\prime \prime}, \ell\right)$ translate to conic sections of the cone $C$ on the perp of $\ell^{D}$, and the two elements of $\operatorname{Tr}(b, \ell) \cap \operatorname{Tr}\left(b^{\prime \prime}, \ell\right)$ give us the two points of the secant line $s$ to the conic given by the intersection of the perps of the points $b^{D}$ and $\left(b^{\prime \prime}\right)^{D}$ dual to $b$ and $b^{\prime \prime}$. Let $\pi_{2}$ and $\pi_{3}$ be the conics arising from taking the perps of the lines $\left\langle b^{D},\left(b^{\prime}\right)^{D}\right\rangle$ and $\left\langle\left(b^{\prime}\right)^{D},\left(b^{\prime \prime}\right)^{D}\right\rangle$. Then by Lemma 2.2 $C$ meets $\pi_{2}$ and $\pi_{3}$ in non-degenerate lines of different types. This means that the perp of $\ell^{D}$ must meet one of $\pi_{2}$ and $\pi_{3}$ in a secant line of $\mathrm{Q}(4, q)$, which is equivalent to $\operatorname{Tr}\left(b^{\prime}, \ell\right)$ meeting either $\operatorname{Tr}(b, \ell)$ or $\operatorname{Tr}\left(b^{\prime \prime}, \ell\right)$ in two elements. Therefore $\equiv_{\ell}$ is transitive, and hence, an equivalence relation.

Now for an arbitrary pair of distinct elements $b, b^{\prime} \in \mathcal{B}$, we know from Lemma 2.1 that the cardinality of $\operatorname{Tr}(b, \ell) \cap \operatorname{Tr}\left(b^{\prime}, \ell\right)$ is 0 or 2 . So the number of elements of $\mathcal{B}$ which are not equivalent to $b$ under $\equiv_{\ell}$ is $(q+1) / 2$. Therefore, the size of the equivalence class of $b$ is also $(q+1) / 2$ and we have completed the proof.

\section{A construction of hemisystems of flock generalised quadrangles}

Construction 3.1. Consider a flock generalised quadrangle $\mathcal{K}(\mathcal{O})$ in the Knarr model with base point $P$ and null polarity $\perp$. Fix a totally isotropic line $\ell$ in $P^{\perp}$ that is disjoint from every element of $\mathcal{O}$. Suppose there is a partition of $\mathcal{O}$ into two halves $\mathcal{O}^{+}, \mathcal{O}^{-}$. For a subset $\mathcal{S}$ of the totally isotropic planes on $\ell$, let $\mathcal{L}_{\mathcal{S}}^{ \pm}$be the line $\$^{4}$ of $\mathcal{K}(\mathcal{O})$ that meet some element of $\mathcal{O}^{ \pm}$in a line, and which meet some element of $\mathcal{S}$ in a point. For any point $X \notin P^{\perp}$ and $\pi \in \mathcal{O}$, define $X^{\pi}$ by

$$
X^{\pi}:=\left\langle\ell, \ell^{\perp} \cap\left\langle X, X^{\perp} \cap \pi\right\rangle\right\rangle .
$$

Note that any $X^{\pi}$ is a totally isotropic plane on $\ell$.

\footnotetext{
${ }^{4}$ Recall that the lines of $\mathcal{K}(\mathcal{O})$ are each totally isotropic planes of $\mathrm{W}(5, q)$.
} 
Theorem 3.2. Let $\mathcal{S}$ be a subset of size $(q-1) / 2$ of the totally isotropic planes on $\ell$ except $\langle P, \ell\rangle$, and let $\mathcal{S}^{c}$ be the complementary set of $(q+1) / 2$ planes on $\ell$ (except $\left.\langle P, \ell\rangle\right)$. In Construction 3.1, the set

$$
\mathcal{O}^{+} \cup \mathcal{L}_{\mathcal{S}}^{+} \cup \mathcal{L}_{\mathcal{S}^{c}}^{-}
$$

of lines of $\mathcal{K}(\mathcal{O})$ is a hemisystem if and only if the following regularity condition holds for any point $X \notin P^{\perp}, \ell^{\perp}$ :

$$
\left|\left\{\pi \in \mathcal{O}^{+} \mid X^{\pi} \in \mathcal{S}\right\}\right|=\left|\left\{\pi \in \mathcal{O}^{-} \mid X^{\pi} \in \mathcal{S}\right\}\right| .
$$

Proof. For each point $X$ not in $P^{\perp}$, we can associate a vector $\tilde{X}$ as follows:

$$
\tilde{X}:=\left[a, a^{c}\right], \quad a=\left|\left\{\pi \in \mathcal{O} \mid X^{\pi} \in \mathcal{S}\right\}\right|, \quad a^{c}=\left|\left\{\pi \in \mathcal{O} \quad \mid X^{\pi} \in \mathcal{S}^{c}\right\}\right| .
$$

This allows us to partition the points of $\mathcal{K}(\mathcal{O})$ so that we can easily construct a tactical decomposition matrix as follows. Consider the matrix whose columns are indexed by $\mathcal{O}^{+}, \mathcal{L}_{\mathcal{S}}^{+}, \mathcal{L}_{\mathcal{S}^{c}}^{+}, \mathcal{L}_{\mathcal{S}}^{-}, \mathcal{L}_{\mathcal{S}^{c}}^{-}$, and whose rows are indexed by a partition of the points of $\mathcal{K}(\mathcal{O})$ which we describe as follows. The single element $P$ is one part of the partition, and the points of type (ii) split into two parts depending if the given point is in some element of $\mathcal{O}^{+}$or $\mathcal{O}^{-}$. (Note that such a point cannot belong to two elements of $\mathcal{O}$ ). For the points of type (i), those in $\ell^{\perp}$ are partitioned by $\mathcal{S}$ and $\mathcal{S}^{c}$. For those outside of $\ell^{\perp}$, we partition the points $X$ according to their vector value $\tilde{X}$.

To show that $\mathcal{O}^{+} \cup \mathcal{L}_{\mathcal{S}}^{+} \cup \mathcal{L}_{\mathcal{S}^{c}}^{-}$is a hemisystem, we need to show that the corresponding columns in this matrix add to the constant column-vector with each entry equal to $(q+1) / 2$. The initial $1+2+2$ rows of the table can be filled in easily, and we then have

\begin{tabular}{l|cc|cccc} 
& $\mathcal{O}^{+}$ & $\mathcal{O}^{-}$ & $\mathcal{L}_{\mathcal{S}}^{+}$ & $\mathcal{L}_{\mathcal{S}^{c}}^{+}$ & $\mathcal{L}_{\mathcal{S}}^{-}$ & $\mathcal{L}_{\mathcal{S}^{c}}^{-}$ \\
\hline$P$ & $\frac{1}{2}(q+1)$ & $\frac{1}{2}(q+1)$ & 0 & 0 & 0 & 0 \\
Point (i) in elt. $\mathcal{S}$ & 0 & 0 & $\frac{1}{2}(q+1)$ & 0 & $\frac{1}{2}(q+1)$ & 0 \\
Point (i) in elt. $\mathcal{S}^{c}$ & 0 & 0 & 0 & $\frac{1}{2}(q+1)$ & 0 & $\frac{1}{2}(q+1)$ \\
Point (ii) in elt. $\mathcal{O}^{+}$ & 1 & 0 & $\frac{1}{2}(q-1)$ & $\frac{1}{2}(q+1)$ & 0 & 0 \\
Point (ii) in elt. $\mathcal{O}^{-}$ & 0 & 1 & 0 & 0 & $\frac{1}{2}(q-1)$ & $\frac{1}{2}(q+1)$ \\
\hline Point (i) $X$ not in $\ell^{\perp}$, & 0 & 0 & $?$ & $?$ & $?$ & $?$ \\
and $\tilde{X}=\left[a, a^{c}\right]$ & & & & & &
\end{tabular}

So now we are left with the submatrix defined by the points of type (i) that are not in $\ell^{\perp}$. Let us consider one row of this submatrix, representing those points $X$ which have a constant value for $\tilde{X}$, say $\left[a, a^{c}\right]$. First notice that $a+a^{c}=q+1$ and hence by the regularity condition of the hypothesis, we know that the corresponding row of the matrix is

$$
\begin{array}{c|cc|cccc} 
& \mathcal{O}^{+} & \mathcal{O}^{-} & \mathcal{L}_{\mathcal{S}}^{+} & \mathcal{L}_{\mathcal{S}^{c}}^{+} & \mathcal{L}_{\mathcal{S}}^{-} & \mathcal{L}_{\mathcal{S}^{c}}^{-} \\
\hline X \notin \ell^{\perp}, \tilde{X}=\left[a, a^{c}\right] & 0 & 0 & \frac{1}{2} a & \frac{1}{2} a^{c} & \frac{1}{2} a & \frac{1}{2} a^{c}
\end{array}
$$

It therefore follows that $\mathcal{O}^{+} \cup \mathcal{L}_{\mathcal{S}}^{+} \cup \mathcal{L}_{\mathcal{S}^{c}}^{-}$is a hemisystem.

Corollary 3.3. Suppose in Construction 3.1, that for all $X \notin P^{\perp}, \ell^{\perp}$,

$$
\left\{X^{\pi} \mid \pi \in \mathcal{O}^{+}\right\}=\left\{X^{\pi} \mid \pi \in \mathcal{O}^{-}\right\} .
$$

Then the set $\mathcal{O}^{+} \cup \mathcal{L}_{\mathcal{S}}^{+} \cup \mathcal{L}_{\mathcal{S}^{c}}^{-}$of lines of $\mathcal{K}(\mathcal{O})$ is a hemisystem for any choice of $\mathcal{S}$ with $|\mathcal{S}|=(q-1) / 2$.

The objective in the remainder of this section is to find a method for finding a partition of $\mathcal{O}$ and a line $\ell$ such that the condition of Corollary 3.3 is satisfied.

Remark 3.4. It is not known whether every hemisystem arising from Theorem 3.2 must also satisfy the stronger condition of the above corollary.

Now we look to the generalised quadrangle $\mathrm{W}(3, q)$ and certain totally isotropic lines which respect a partition of the BLT-set $\mathcal{B}$. Recall from Section 2.2 that any line of $\mathrm{W}(3, q)$ not in $\mathcal{B}$ must be concurrent with 0 or 2 elements of $\mathcal{B}$. So in particular, if we fix a point $X$ not in any element of $\mathcal{B}$, then the map

$$
b \mapsto\left\langle X, b \cap X^{\perp}\right\rangle
$$

from $\mathcal{B}$ to lines on $X$ is two-to-one (see also [1, Lemma 3.4]).

Definition 3.5. Let $\mathcal{B}^{+}$and $\mathcal{B}^{-}$be a partition of a BLT-set $\mathcal{B}$ of lines of $\mathrm{W}(3, q)$. Then a totally isotropic line $\ell$ not meeting any element of $\mathcal{B}$ is compatible with the partition $\left\{\mathcal{B}^{+}, \mathcal{B}^{-}\right\}$if for all $X \in \ell$, we have $\left\{\left\langle X, b \cap X^{\perp}\right\rangle \mid b \in \mathcal{B}^{+}\right\}=\left\{\left\langle X, b \cap X^{\perp}\right\rangle \mid b \in \mathcal{B}^{-}\right\}$.

We can identify when Corollary 3.3 holds by just looking to the quotient symplectic space $P^{\perp} / P$. 
Lemma 3.6. Consider a flock generalised quadrangle $\mathcal{K}(\mathcal{O})$ in the Knarr model with base point $P$ and null polarity $\perp$. Fix a totally isotropic line $\ell$ in $P^{\perp}$ that is disjoint from every element of $\mathcal{O}$. Suppose there is a partition of $\mathcal{O}$ into two halves $\mathrm{O}^{+}$and $\mathcal{O}^{-}$. Then the following two conditions are equivalent:

(i) For all $E \notin P^{\perp}, \ell^{\perp}$, we have $\left\{E^{\pi} \mid \pi \in \mathcal{O}^{+}\right\}=\left\{E^{\pi} \mid \pi \in \mathcal{O}^{-}\right\}$.

(ii) The line $\langle P, \ell\rangle / P$ of the quotient symplectic space $P^{\perp} / P$ is compatible with the following partition of the projection of $\mathcal{O}$ to $P^{\perp} / P$ :

$$
\mathcal{O}^{+} / P:=\left\{\pi / P \mid \pi \in \mathcal{O}^{+}\right\}, \quad \mathcal{O}^{-} / P:=\left\{\pi / P \mid \pi \in \mathcal{O}^{-}\right\}
$$

Proof. Let $E \notin P^{\perp}, \ell^{\perp}$. We will show that the two sets of objects are in bijective correspondence, and to do this, we break the proof down into steps. These steps require us to define six sets of objects as follows. Let:

- Planes $(P)$ be the set of planes on $P$ not meeting $\ell$;

- $\operatorname{Lines}\left(P^{\perp} \cap\left\langle\ell^{\perp}, E\right\rangle\right)$ be the set of lines on $P$ in the 3 -space $P^{\perp} \cap\left\langle\ell^{\perp}, E\right\rangle$, meeting $\langle P, \ell\rangle$ in $P$;

- Planes $\left(P^{\perp} \cap\left\langle\ell^{\perp}, E\right\rangle\right)$ be the set of planes on $P$ inside $P^{\perp} \cap\left\langle\ell^{\perp}, E\right\rangle$ meeting $\ell$ in a point;

- Planes $(E)$ be the set of planes on $E$ not meeting $\langle P, \ell\rangle$;

- Points $\left(E^{\perp} \cap \ell^{\perp}\right)$ be the set of points of the plane $E^{\perp} \cap \ell^{\perp}$ not in $P^{\perp}$;

- Planes $(\ell)$ be the set of planes on $\ell$, not on $P$.

In fact, we will eventually establish that the diagram below commutes, and it will serve as a guide to showing that $\Phi$ on the bottom line is bijective by proving that particular maps are surjections whose preimages yield regular partitions:

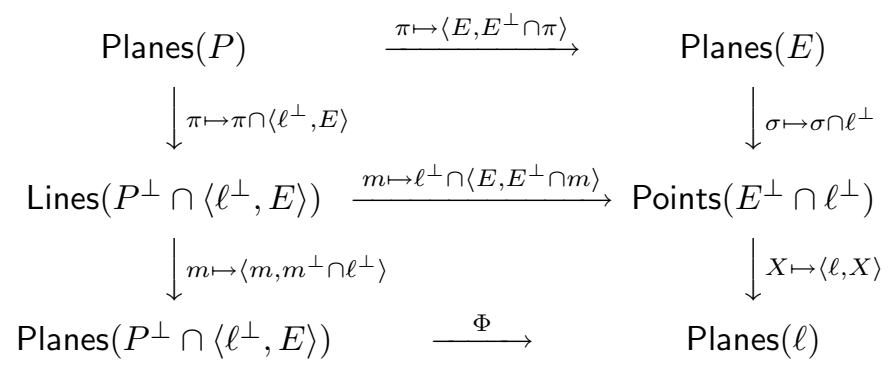

where $\Phi$ is defined by

$$
\Phi:\left\langle m, m^{\perp} \cap \ell^{\perp}\right\rangle \rightarrow\left\langle\ell, \ell^{\perp} \cap\left\langle E, E^{\perp} \cap \pi_{m}\right\rangle\right\rangle, \quad\left(\pi_{m}:=\left\langle m, m^{\perp} \cap \ell^{\perp}\right\rangle\right) .
$$

Let us look at these maps in turn. It is not difficult to see that the map $\pi \mapsto \pi \cap\left\langle\ell^{\perp}, E\right\rangle$ is a $q$-to-one surjective map from Planes $(P)$ onto $\operatorname{Lines}\left(P^{\perp} \cap\left\langle\ell^{\perp}, E\right\rangle\right)$, as each element of $\operatorname{Lines}\left(P^{\perp} \cap\left\langle\ell^{\perp}, E\right\rangle\right)$ is incident with $q$ elements of Planes $(P)$. The map $m \mapsto\left\langle m, m^{\perp} \cap \ell^{\perp}\right\rangle$ is also a $q$-to-one surjective map, but this time from Lines $\left(P^{\perp} \cap\left\langle\ell^{\perp}, E\right\rangle\right)$ onto $\operatorname{Planes}\left(P^{\perp} \cap\left\langle\ell^{\perp}, E\right\rangle\right)$, since each element of Planes $\left(P^{\perp} \cap\left\langle\ell^{\perp}, E\right\rangle\right)$ contains $q$ elements of $\operatorname{Lines}\left(P^{\perp} \cap\left\langle\ell^{\perp}, E\right\rangle\right)$. So the composition of these maps, which we see in the diagram as "down from the top left to the bottom left", is a $q^{2}$-to-one surjective map from Planes $(P)$ onto Planes $\left(P^{\perp} \cap\left\langle\ell^{\perp}, E\right\rangle\right)$. If we can show that the map $\pi \mapsto\left\langle\ell, \ell^{\perp} \cap\left\langle E, E^{\perp} \cap \pi\right\rangle\right\rangle$ is a $q^{2}$-to-one surjection, then $\Phi$ will be well-defined and bijective. Now the map $\pi \mapsto\left\langle E, E^{\perp} \cap \pi\right\rangle$ is injective since if $\left\langle E, E^{\perp} \cap \pi_{1}\right\rangle=\left\langle E, E^{\perp} \cap \pi_{2}\right\rangle$ for two distinct planes $\pi_{1}$ and $\pi_{2}$, then $E^{\perp} \cap \pi_{1}$ and $E^{\perp} \cap \pi_{2}$ are coplanar and hence meet in at least a point, which implies that $P \in E^{\perp}$; a contradiction. Now we work our way down the right hand side of the diagram. The map $\sigma \mapsto \sigma \cap \ell^{\perp}$ is clearly a $q$-to-one surjection (as every point of Points $\left(E^{\perp} \cap \ell^{\perp}\right)$ is incident with $q$ elements of Planes $(E)$ ), and the map $X \mapsto\langle\ell, X\rangle$ from Points $\left(E^{\perp} \cap \ell^{\perp}\right)$ to Planes $(\ell)$ is also a $q$-to-one surjection. So we see that the composition of the maps traversing downwards on the right-hand side of the diagram is a $q^{2}$-to-one surjection. Therefore it follows that the left-to-right maps in the diagram are each bijections, and in particular, $\Phi$ is a bijection.

So we have established that condition (i) of the hypothesis is equivalent to the condition that for all $E \notin P^{\perp}, \ell^{\perp}$ we have

$$
\left\{\left\langle m, m^{\perp} \cap \ell^{\perp}\right\rangle \mid m=\pi \cap\left\langle\ell^{\perp}, E\right\rangle, \pi \in \mathcal{O}^{+}\right\}=\left\{\left\langle m, m^{\perp} \cap \ell^{\perp}\right\rangle \mid m=\pi \cap\left\langle\ell^{\perp}, E\right\rangle, \pi \in \mathcal{O}^{-}\right\} .
$$

Now we project by $P$ to the symplectic space $P^{\perp} / P$ (which is isomorphic to $\mathrm{W}(3, q)$ ). The image of $\mathcal{O}$ under the projection is a BLT-set of lines of $P^{\perp} / P$. The plane $\langle P, \ell\rangle$ is mapped to a line which does not meet any element of the BLT-set of lines. If $\pi \in \mathcal{O}$, then the line $m=\pi \cap\left\langle\ell^{\perp}, E\right\rangle$ projects to the unique point on $\pi / P$ which is collinear to the point $\left(\ell \cap E^{\perp}\right) / P$ on $\langle P, \ell\rangle / P$. 


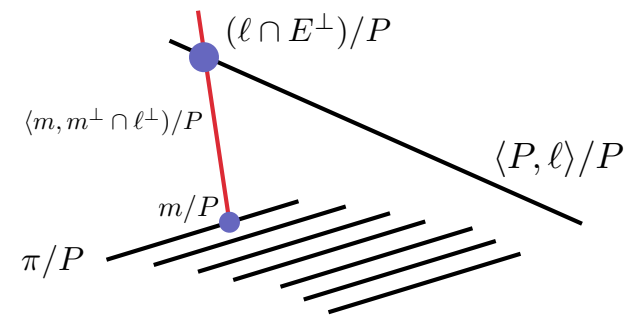

Figure 2: The projection to $P^{\perp} / P$.

The choice of $E$ corresponds to the choice of the point on $\langle P, \ell\rangle / P$. So the condition (1) is equivalent to having, for all points $X$ on the line $\langle P, \ell\rangle / P$,

$$
\left\{\left\langle X, b \cap X^{\perp}\right\rangle \mid b \in \mathcal{O}^{+} / P\right\}=\left\{\left\langle X, b \cap X^{\perp}\right\rangle \mid b \in \mathcal{O}^{-} / P\right\},
$$

that is, $\langle P, \ell\rangle / P$ is compatible with the partition $\left\{\mathcal{O}^{+} / P, \mathcal{O}^{-} / P\right\}$.

The remaining ingredient for the proof of Theorem 1.1 is the following:

Theorem 3.7. Consider a set $\mathcal{O}$ of totally isotropic planes of $\mathrm{W}(5, q)$ each incident with a point $P$ such that

$$
\mathcal{B}:=\{\pi / P \mid \pi \in \mathcal{O}\}
$$

is a BLT-set of lines of the quotient symplectic space $P^{\perp} / P \cong \mathrm{W}(3, q)$. Suppose, furthermore, that we have a line $\ell$ of $P^{\perp} / P$ not meeting any element of $\mathcal{B}$, and let $\equiv_{\ell}$ be the binary relation on $\mathcal{B}$ given in Definition 2.3. Then $\ell$ is compatible with the equivalence classes of $\equiv_{\ell}$.

Proof. Let $\mathcal{B}^{+}$and $\mathcal{B}^{-}$be the two equivalence classes of $\equiv_{\ell}$. Let $E$ be a point on $\ell$, and let $m$ be a line on $E$ concurrent with two distinct elements $b$ and $b^{\prime}$ of $\mathcal{B}$. Since $m$ is both an element of $\operatorname{Tr}(b, \ell)$ and

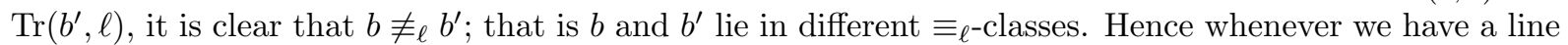
$m$ concurrent with $\ell$ and concurrent with two elements $b$ and $b^{\prime}$ of $\mathcal{B}$, then $m=\left\langle E, b \cap E^{\perp}\right\rangle=\left\langle E, b^{\prime} \cap E^{\perp}\right\rangle$ where $E=m \cap \ell$, and we can assume that $b \in \mathcal{B}^{+}$and $b^{\prime} \in \mathcal{B}^{-}$. So it follows, that for all $E \in \ell$, we have $\left\{\left\langle E, b \cap E^{\perp}\right\rangle \mid b \in \mathcal{B}^{+}\right\}=\left\{\left\langle E, b^{\prime} \cap E^{\perp}\right\rangle \mid b^{\prime} \in \mathcal{B}^{-}\right\}$. Therefore $\ell$ is compatible with $\left\{\mathcal{B}^{+}, \mathcal{B}^{-}\right\}$.

So together with Corollary 3.3 and Lemma 3.6, we obtain a hemisystem of the associated flock generalised quadrangle and hence we have proved Theorem 1.1

\section{Remark 3.8.}

(1) The hemisystem one obtains from Corollary 3.3 depends on the choice of a set $\mathcal{S}$ of $(q-1) / 2$ totally isotropic planes on a line $\ell\left(\right.$ in $P^{\perp}$ disjoint from every element of $\mathcal{O}$ ). For a set $\mathcal{O}$ and line $\ell$ as above, we can obtain different hemisystems by the choice of $\mathcal{S}$, which we explore further in [4].

(2) To our knowledge, the only possible partition $\left\{\mathcal{O}^{+}, \mathcal{O}^{-}\right\}$that works for a given line $\ell$ is the one arising from the equivalence relation $\equiv_{\ell}$. We have found no counter-examples in small flock generalised quadrangles.

\section{The Cossidente-Penttila hemisystems}

As we mentioned in the introduction, Cossidente and Penttila 12 produced for each odd prime power $q$, a hemisystem of $\mathrm{H}\left(3, q^{2}\right)$ admitting $\mathrm{P} \Omega^{-}(4, q)$. Their construction can be summarised as follows. Consider an elliptic quadric $\mathcal{E} \cong \mathrm{Q}^{-}(3, q)$ whose defining polarity commutes with the polarity defining a fixed Hermitian variety $\mathrm{H}\left(3, q^{2}\right)$. We then see that all the $q^{2}+1$ singular points of $\mathcal{E}$ are totally isotropic points of $\mathrm{H}\left(3, q^{2}\right)$, and each totally isotropic line of $\mathrm{H}\left(3, q^{2}\right)$ meets $\mathcal{E}$ in either no points or one point (see the first line of the proof of [12, Proposition 2.3]). We may call such lines external and tangent respectively. The key ingredient is the action of the perfect group $\mathrm{P} \Omega^{-}(4, q)$ on the lines of $\mathrm{H}\left(3, q^{2}\right)$; it has two orbits on tangents and two orbits on externals (this follows from the proof of [12, Theorem 3.1]). Take one of each and their union is a hemisystem admitting $\mathrm{P} \Omega^{-}(4, q)$. In fact, it is clear that this is the only way one can obtain a hemisystem admitting this group $\mathrm{P} \Omega^{-}(4, q)$ stabilising $\mathcal{E}$. We will show in this section that the Cossidente-Penttila hemisystems can be constructed from Theorem 3.1, and we first interpret the original construction in the following way: 
Lemma 4.1. Let $q$ be an odd prime power and consider an elliptic quadric $\mathcal{E}:=\mathrm{Q}^{-}(3, q)$ and Hermitian variety $\mathrm{H}\left(3, q^{2}\right)$ whose defining polarities commute. Let $G$ be the subgroup $\mathrm{P} \Omega^{-}(4, q)$ stabilising $\mathcal{E}$ and let $\Omega$ be one of the two orbits of size $q^{2}\left(q^{2}-1\right) / 2$ of $G$ on totally isotropic lines of $\mathrm{H}\left(3, q^{2}\right)$ external to $\mathcal{E}$. Fix a point $P \in \mathcal{E}$ and let $\mathcal{P}^{+}$and $\mathcal{P}^{-}$be the two orbits of $G_{P}$ on lines on $P$. Let $\mathcal{M}^{+}$be the set of lines of $\Omega$ which meet a unique element of $\mathcal{P}^{+}$in a point. Let $\mathcal{M}^{-}$be comprised of the set of lines of $\Omega$ which meet a unique element of $\mathcal{P}^{-}$in a point, together with the tangent lines which meet a unique element of $\mathcal{P}^{-}$in a point. Then

$$
\mathcal{P}^{+} \cup \mathcal{M}^{+} \cup \mathcal{M}^{-}
$$

is a hemisystem of $\mathrm{H}\left(3, q^{2}\right)$ admitting $G$, and hence, is equivalent to a Cossidente-Penttila hemisystem.

Proof. Let $\mathcal{M}_{T}^{-}$be the tangent lines of $\mathcal{M}^{-}$and let $\mathcal{M}_{E}^{-}$be the external lines of $\mathcal{M}^{-}$. We will show that $\mathcal{P}^{+} \cup \mathcal{M}_{T}^{-}$is a $G$-orbit on tangents and that $\mathcal{M}_{E}^{-} \cup \mathcal{M}^{+}$is a $G$-orbit on externals. Clearly $\Omega=\mathcal{M}_{E}^{-} \cup \mathcal{M}^{+}$as every line of $\Omega$ must meet $P^{\perp}$ and hence is concurrent with some element of $\mathcal{P}^{+}$or $\mathcal{P}^{-}$. Also, $\mathcal{M}_{T}^{-}$is an orbit of $G_{P}$ on tangents. Let $Y$ be a point of $\mathrm{H}\left(3, q^{2}\right)$ not in $\mathcal{E}$ but "nearby" to $\mathcal{E}$, that is, there is some point $X$ of $\mathcal{E}$ such that $Y$ is collinear with $X$ in $\mathrm{H}\left(3, q^{2}\right)$. Now there are $q^{2}\left(q^{2}+1\right)(q+1) / 2$ "nearby" points in total (by the last paragraph of the proof of [12, Proposition 2.2]), there are $q^{2}+1$ points of $\mathcal{E}$ and any point of $\mathcal{E}$ is collinear to $q^{2}(q+1)$ other points of $\mathrm{H}\left(3, q^{2}\right)$. So there are exactly two lines on $Y$ which are tangent to $\mathcal{E}$, and these two lines are in different $G$-orbits. Now suppose we have an element $m \in \mathcal{M}_{T}^{-}$. Then $m$ is concurrent with a unique line on $P$ in a point $Y$, say, and the two tangents on $Y$ (namely $m$ and $\langle P, Y\rangle$ ) are in different $G$-orbits and $\langle P, Y\rangle \in \mathcal{P}^{-}$by the definition of $\mathcal{M}_{T}^{-}$. Therefore, $\mathcal{P}^{+} \cup \mathcal{M}_{T}^{-}$is contained in a $G$-orbit, but since it has cardinality half the number of tangents, $\mathcal{P}^{+} \cup \mathcal{M}_{T}^{-}$is a $G$-orbit on tangents. So we see that $\mathcal{P}^{+} \cup \mathcal{M}^{+} \cup \mathcal{M}^{-}$is the union of a $G$-orbit on externals and a $G$-orbit on tangents, and so it follows that it is equivalent to a Cossidente-Penttila hemisystem.

4.1. The Knarr model is a generalisation of field reduction. One can view the Knarr model of a flock generalised quadrangle as a generalisation of the field reduction of $\mathrm{H}\left(3, q^{2}\right)$ to $\mathrm{W}(7, q)$, and we will take some time now to explain how this works. Let $\Phi$ be the map from $\mathrm{H}\left(3, q^{2}\right)$ to $\mathrm{W}(7, q)$ obtained by identifying $\mathrm{GF}\left(q^{2}\right)$ with $\mathrm{GF}(q)^{2}$ such that the four-dimensional vector space over $\operatorname{GF}\left(q^{2}\right)$ becomes an eight-dimensional vector space over $\mathrm{GF}(q)$. Let $\xi$ be an element of $\mathrm{GF}\left(q^{2}\right)$ not in $\mathrm{GF}(q)$ whose relative trace $\mathrm{T}_{q^{2} \rightarrow q}(\xi)=\xi+\xi^{q}$ is zero. Then the following form is bilinear and alternating over $\mathrm{GF}(q)$ :

$$
B(u, v)=\mathrm{T}_{q^{2} \rightarrow q}(\xi \cdot \beta(u, v))
$$

where $\beta$ is the original Hermitian form over $\operatorname{GF}\left(q^{2}\right)$. Under this correspondence points go to lines, lines go to solids, and so forth. Fix a point $P$ of $\mathrm{H}\left(3, q^{2}\right)$ and the $q+1$ Hermitian lines $\mathcal{P}$ incident with $P$. Then under $\Phi$, we obtain a symplectic line $P^{\Phi}$ of $\mathrm{W}(7, q)$ and $q+1$ symplectic solids $\mathcal{P}^{\Phi}$. Now let $\Pi$ be a hyperplane of $\mathrm{PG}(7, q)$ such that $\Pi^{\perp}$ is a point on $P^{\Phi}$. Consider the following projection map from totally isotropic subspaces of $\mathrm{W}(7, q)$ to totally isotropic subspaces of the quotient polar space $\Pi / \Pi^{\perp} \cong \mathrm{W}(5, q)$ :

$$
\rho: U \mapsto\left(\Pi \cap\left\langle\Pi^{\perp}, U\right\rangle\right) / \Pi^{\perp} .
$$

The composition of $\Phi$ and $\rho$ then gives us the Knarr model of $\mathrm{H}\left(3, q^{2}\right)$ :

\begin{tabular}{l|l} 
Objects in $\mathrm{H}\left(3, q^{2}\right)$ & Objects in $\mathrm{W}(5, q)$ \\
\hline & \\
Lines on $P$ & Point $P^{\Phi \rho}$ of $\mathrm{W}(5, q)$ \\
Points collinear with $P$ & BLT-set of totally isotropic planes on $P^{\Phi \rho}$ \\
Points not collinear with $P$ & Lines incident with some BLT-set element, not incident with $P^{\Phi \rho}$ \\
Lines not on $P$ & Points not collinear with $P^{\Phi \rho}$ \\
& Planes, not on $P^{\Phi \rho}$, which meet some BLT-set element in a line \\
\hline
\end{tabular}

Table 2: The Knarr model of $\mathrm{H}\left(3, q^{2}\right)$ obtained via field reduction and projection. 
4.2. The elliptic quadric in $\mathrm{W}(7, q)$. It will be useful in understanding and proving Theorem 4.2 to geometrically describe the image of the elliptic quadric $\mathcal{E}$ in $\mathrm{W}(7, q)$. Let $V$ be a 4-dimensional vector space over GF $\left(q^{2}\right)$ with $q$ odd, equipped with a non-degenerate Hermitian form $\beta$. Let $\left\{v_{1}, v_{2}, v_{3}, v_{4}\right\}$ be a basis for $V$ such that the determinant of the Gram matrix of $\beta$ with respect to this basis is a nonsquare in $\mathrm{GF}(q)$. (For example, take an orthonormal basis and multiply the first basis element by a primitive element of $\mathrm{GF}\left(q^{2}\right)$.) Let $U$ be the $\mathrm{GF}(q)$-span of $\left\{v_{1}, v_{2}, v_{3}, v_{4}\right\}$. Then $V=U \otimes \mathrm{GF}\left(q^{2}\right)$ and the restriction $\beta_{1}$ of $\beta$ to $U$ is a symmetric bilinear form associated with an elliptic quadratic form. Then $g \in O^{-}(4, q)$ acts on $V$ such that $(u \otimes \lambda)^{g}=u^{g} \otimes \lambda$ for all $g \in O^{-}(4, q), u \in U$ and $\lambda \in \operatorname{GF}\left(q^{2}\right)$. This action preserves the form $\beta$ and so we have an embedding of isometry groups $O^{-}(4, q) \leqslant U\left(4, q^{2}\right)$.

Now recall from Section 4.1 that $V$ is also an 8-dimensional vector space over $\mathrm{GF}(q)$ and is equipped with a non-degenerate alternating form $B=\mathrm{T}_{q^{2} \rightarrow q}(\xi \cdot \beta)$ where $\mathrm{T}_{q^{2} \rightarrow q}(\xi)=0$. Now for $u \otimes \lambda, w \otimes \mu \in$ $U \otimes \operatorname{GF}\left(q^{2}\right)$, we have

$$
\begin{aligned}
B(u \otimes \lambda, w \otimes \mu) & =\mathrm{T}_{q^{2} \rightarrow q}(\xi \cdot \beta(u \otimes \lambda, w \otimes \mu)) \\
& =\mathrm{T}_{q^{2} \rightarrow q}\left(\xi \lambda \mu^{q} \cdot \beta(u \otimes 1, w \otimes 1)\right) \\
& =\beta_{1}(u, w) \mathrm{T}_{q^{2} \rightarrow q}\left(\xi \lambda \mu^{q}\right)
\end{aligned}
$$

since $\beta_{1}(u, w) \in \mathrm{GF}(q)$. Moreover, $\beta_{2}(\lambda, \mu)=\mathrm{T}_{q^{2} \rightarrow q}\left(\xi \lambda \mu^{q}\right)$ is an alternating form on the 2-dimensional space $W=\mathrm{GF}\left(q^{2}\right)$ as $\lambda \mu^{q}$ defines a Hermitian form on $W$. From now on we consider $W$ as a 2-dimensional vector space over $\mathrm{GF}(q)$ such that $V=U \otimes W$. Also, given the symmetric form $\beta_{1}$ on $U$ and the alternating form $\beta_{2}$ on $W$ we have

$$
B\left(u_{1} \otimes w_{1}, u_{2} \otimes w_{2}\right)=\beta_{1}\left(u_{1}, u_{2}\right) \beta_{2}\left(w_{1}, w_{2}\right) .
$$

Then the central product $O^{-}(4, q) \circ \operatorname{Sp}(2, q)$ acts naturally on $V$ via $(u \otimes w)^{(g, h)}=u^{g} \otimes w^{h}$ for all $g \in O^{-}(4, q), h \in \mathrm{Sp}(2, q), u \in U$ and $w \in W$, and this action preserves the form $B$.

4.3. A Knarr model of the Cossidente-Penttila hemisystems. By the following result, we see how one can obtain the Cossidente-Penttila hemisystems via Theorem 3.1. First we summarise in a table the notation which we are using in this section:

\begin{tabular}{ll}
\hline Object & Description \\
\hline $\mathcal{E}$ & an elliptic quadric embedded in $\mathrm{H}\left(3, q^{2}\right)$ \\
$G$ & subgroup $\mathrm{P}^{-}(4, q)$ stabilising $\mathcal{E}$ \\
$\Omega$ & a $G$-orbit on totally isotropic lines of $\mathrm{H}\left(3, q^{2}\right)$ external to $\mathcal{E}$ \\
$\mathcal{P}$ & lines on $P$ \\
$\mathcal{P}^{+}, \mathcal{P}^{-}$ & G-orbits on $\mathcal{P}$ \\
$\mathcal{M}^{+}$ & set of lines of $\Omega$ which meet an element of $\mathcal{P}^{+}$in a point \\
$\mathcal{M}^{-}$ & set of lines of $\Omega$ which meet an element of $\mathcal{P}^{-}$in a point, together with the tangent lines \\
& which an element of $\mathcal{P}^{-}$in a point \\
$\mathcal{O}$ & $\mathcal{P}^{\Phi \rho}$ \\
$\mathcal{O}^{+}$ & $\left(\mathcal{P}^{+}\right)^{\Phi \rho}$ \\
$\mathcal{O}^{-}$ & $\left(\mathcal{P}^{-}\right)^{\Phi \rho}$ \\
\hline
\end{tabular}

Theorem 4.2. Consider the $q^{2}+1$ points $\mathcal{E}^{\Phi \rho}$ of $\mathrm{W}(5, q)$ obtained by field reduction to $\mathrm{W}(7, q)$ and projection to $\mathrm{W}(5, q)$ as described above. Then $\mathcal{E}^{\Phi \rho}$ spans a solid whose image under the null polarity is a totally isotropic line $\ell$ of $\mathrm{W}(5, q)$. For a subset $\mathcal{S}$ of the totally isotropic planes on $\ell$, let $\mathcal{L}_{\mathcal{S}}^{ \pm}$be the totally isotropic planes of $\mathrm{W}(5, q)$ that meet some element of $\mathcal{O}^{ \pm}$in a line, and that meet some element of $\mathcal{S}$ in a point. Let $\mathcal{R}$ be the set of (t.i.) planes on $\ell$ that meet some element of $\left(\mathcal{M}^{+}\right)^{\Phi \rho}$ in a point. Then $\mathcal{R}$ has size $(q-1) / 2,\left(\mathcal{M}^{+}\right)^{\Phi \rho}=\mathcal{L}_{\mathcal{R}}^{+}$and $\left(\mathcal{M}^{-}\right)^{\Phi \rho}=\mathcal{L}_{\mathcal{R}^{c}}^{-}$.

ProOF. First we show that $\mathcal{E}^{\Phi \rho}$ spans a solid whose perp is a totally isotropic line of $\mathrm{W}(5, q)$. Recall from the discussion in Section 4.2 that we can write our 8-dimensional vector space $V$ as $U \otimes W$ where $U$ carries a symmetric bilinear form of minus type (and is 4-dimensional) and $W$ carries an alternating bilinear form (and is 2-dimensional) such that the "product" of these two forms defines a non-degenerate alternating bilinear form on $V$. Let $u \in U$ be a totally singular vector with respect to the elliptic form on $U$. Then $\langle u\rangle \otimes W$ is a totally isotropic line in $V$ with respect to $B$, and so we will identify $P^{\Phi \rho}$ with $(\langle u\rangle \otimes W)^{\rho}$. Fix $w \in W$ and let $X=\langle u \otimes w\rangle$. Then $X^{\perp}$ is a hyperplane containing $u^{\perp} \otimes W$. We can also find an element $\bar{u} \in U$ such that $\beta(\bar{u}, u) \neq 0$. Then $B(\bar{u} \otimes w, u \otimes w)=\beta_{1}(\bar{u}, u) \beta_{2}(w, w)=0$. Hence $X^{\perp}=\left\langle\bar{u} \otimes w, u^{\perp} \otimes W\right\rangle$.

Now we may write the image of our elliptic quadric as

$$
\mathcal{E}^{\Phi}=\left\{\left\langle u^{\prime}\right\rangle \otimes W \mid u^{\prime} \in U, \beta_{1}\left(u^{\prime}, u^{\prime}\right)=0\right\} .
$$


Then for $\left\langle u^{\prime}\right\rangle \otimes W \in \mathcal{E}$ we have

$$
X^{\perp} \cap\left(\left\langle u^{\prime}\right\rangle \otimes W\right)=\left\{\begin{array}{ll}
\left\langle u^{\prime}\right\rangle \otimes W & \text { if } u^{\prime} \in u^{\perp} \\
\left\langle u^{\prime} \otimes w\right\rangle & \text { if } u^{\prime} \notin u^{\perp}
\end{array} .\right.
$$

The only totally singular point in $u^{\perp}$ is $u$, so

$$
\left\langle\left\{X^{\perp} \cap E \mid E \in \mathcal{E}^{\Phi}\right\}\right\rangle=\left\langle u \otimes W, u^{\prime} \otimes w \mid u^{\prime} \notin u^{\perp}\right\rangle=\langle U \otimes w, u \otimes W\rangle,
$$

which is a 4-dimensional projective space whose perp is a totally isotropic plane of $\mathrm{W}(7, q)$. So returning to our model, we can substitute in this argument $X^{\perp}$ for the the hyperplane $\Pi$ in the Knarr model (2) and we see that $\mathcal{E}^{\Phi \rho}$ spans a solid whose perp is a totally isotropic line $\ell$ of $\mathrm{W}(5, q)$.

Now consider the set $\mathcal{R}$ of totally isotropic planes on $\ell$ that meet some element of $\left(\mathcal{M}^{+}\right)^{\Phi \rho}$ in a point. First note that for every line $m$ of $\mathrm{H}\left(3, q^{2}\right)$, we have that $m^{\Phi \rho}$ meets $\ell^{\perp}$ in a point (n.b., $\perp$ now denotes the polarity defining $\mathrm{W}(5, q))$. Moreover, if $m$ is a tangent line to $\mathcal{E}$, then $m^{\Phi \rho} \cap \ell^{\perp} \in \mathcal{E}^{\Phi \rho}$. Now $\mathcal{E}^{\Phi}$ contains the line $P^{\Phi}=\langle u\rangle \otimes W$, and the other lines $\left\langle u^{\prime}\right\rangle \otimes W$ meet the totally isotropic solid $\langle U \otimes w\rangle$ in a point. So $\mathcal{E}^{\Phi \rho}$ consists precisely of the point $P^{\Phi \rho}$ and the points of the totally isotropic plane $\langle U \otimes w\rangle^{\rho}$ which are not on $\ell$. This plane $\langle U \otimes w\rangle^{\rho}$ is incident with $\ell$, so we know that just two of the planes on $\ell$ meet the images of tangent lines under $\Phi \rho$, whereas the remaining $q-1$ planes meet the images of external lines. It is then not difficult to ascertain that half of these $q-1$ planes are the elements of $\mathcal{R}$.

By definition, $\mathcal{O}^{+}$is the image of $\mathcal{P}^{+}$under $\Phi \rho$. Let $m \in \mathcal{M}^{+}$, that is, $m \in \Omega$ and $m$ meets some element of $b \in \mathcal{P}^{+}$in a point $E$. Then $m^{\Phi}$ is a solid meeting the solid $b^{\Phi}$ in the line $E^{\Phi}$. So $m^{\Phi \rho}$ is a plane meeting an element of $\mathcal{O}^{+}$in $E^{\Phi \rho}$, the latter being a line as $E^{\Phi} \leqslant X^{\perp}$. Now $m^{\Phi \rho}$ meets $\ell^{\perp}$ in a point, and so there is a unique totally isotropic plane on $\ell$ which meets $m^{\Phi \rho}$ in a point, namely $\left\langle\ell, \ell^{\perp} \cap m^{\Phi \rho}\right\rangle$. By definition, this plane must be an element of $\mathcal{R}$, and hence, $m^{\Phi \rho} \in \mathcal{L}_{\mathcal{R}}^{+}$. It is not difficult to show that the cardinalities of $\mathcal{M}^{+}$and $\mathcal{L}_{\mathcal{R}}^{+}$are equal to $q^{2}\left(q^{2}-1\right) / 4$ and hence $\left(\mathcal{M}^{+}\right)^{\Phi \rho}=\mathcal{L}_{\mathcal{R}}^{+}$. By a similar argument, we have that $\left(\mathcal{M}^{-}\right)^{\Phi \rho}=\mathcal{L}_{\mathcal{R}^{c}}^{-}$.

Corollary 4.3. The Cossidente-Penttila hemisystem of $\mathrm{H}\left(3, q^{2}\right)$ can be described as $\mathcal{O}^{+} \cup \mathcal{L}_{\mathcal{R}}^{+} \cup \mathcal{L}_{\mathcal{R}^{c}}^{-}$in the Knarr model $\mathcal{K}(\mathcal{O})$ of $\mathrm{H}\left(3, q^{2}\right)$, that is, the Cossidente-Penttila hemisystems occur as examples of output of our Construction 3.1 . 


\section{Appendix: A proof that a hemisystem yields a partial quadrangle}

Let $\mathcal{Q}$ be a generalised quadrangle of order $\left(s, s^{2}\right), s>1$ odd. If $\mathcal{P}$ is the set of points of $\mathcal{Q}$, then the algebra $\mathbb{C} \mathcal{P}$ of functions from $\mathcal{P}$ to $\mathbb{C}$ decomposes into an orthogonal decomposition of three irreducible subspaces $V^{0}, V^{+}$and $V^{-}$, which are in fact eigenspaces for the adjacency matrix of $\mathcal{Q}$ with eigenvalues $s\left(s^{2}+1\right), s-1$ and $-s^{2}-1$ respectively. Moreover, $V^{0}$ is just the subspace of constant functions from $\mathcal{P}$ to $\mathbb{C}$, which is spanned by the all-one function $j$. We will be considering multisets of elements of $\mathcal{P}$, that is, functions from $\mathcal{P}$ to the non-negative integers $\mathbb{N}_{0}$. To make our notation simpler, we will avoid using the symbol "chi" and a subscript for the characteristic function of a multiset, rather we will simply use the multiset itself if it is clear that we are using it as an element of $\mathbb{C P}$.

A weighted tight set is any multiset $v$ of $\mathbb{N}_{0} \mathcal{P}$ such that $v \in V^{0} \bigoplus V^{+}$. Similarly, a weighted m-ovoid is any multiset residing in $V^{0} \bigoplus V^{-}$. An example of a weighted $m$-ovoid is a hemisystem of points of a generalised quadrangle; the dual notion of a hemisystem of lines (see [5, Lemma 1]). The scalar product $A \bullet B$ of two multisets $A$ and $B$ gives us the function $i \mapsto A(i) B(i)$, which one can think of as the generalisation of the intersection of two sets. Therefore, we write $|A|$ for $A \bullet j$. So suppose we have a weighted tight set $v^{+}$and a weighted $m$-ovoid $v^{-}$. Then by orthogonal projection, we see that

$$
v^{+}-\frac{\left|v^{+}\right|}{|\mathcal{P}|} j \in V^{+} \quad \text { and } \quad v^{-}-\frac{\left|v^{-}\right|}{|\mathcal{P}|} j \in V^{-}
$$

and hence these two vectors are orthogonal to one another. This observation then implies that $v^{+}$and $v^{-}$meet in a number of elements determined by their sizes:

$$
v^{+} \bullet v^{-}=\frac{\left|v^{+}\right|\left|v^{-}\right|}{|\mathcal{P}|} .
$$

(Note that $|\mathcal{P}|$ is simply the number $(s+1)\left(s^{3}+1\right)$ of points of $\mathcal{Q}$ ).

Lemma 5.1. Let $x$ and $y$ be two noncollinear points of $\mathcal{Q}$ and consider the multiset $\mathcal{T}$ of points consisting of $\{x, y\}^{\perp}$ and $s$ copies of $x$ and $y$. Then $\mathcal{T}$ is a weighted tight set.

Proof. Consider the function $v:=\left(s^{3}+1\right) \mathcal{T}-(s+1) j$. We will show that $v$ is an eigenvector of the adjacency matrix $A$ with eigenvalue $s-1$. Now we collect a few formulae which are simple to demonstrate with geometric arguments, plus the fact that the size of $\{x, y, z\}^{\perp}$ is $s+1$, where $x, y, z$ are pairwise noncollinear (see [8]):

$$
\begin{aligned}
j A & =s\left(s^{2}+1\right) j, \\
\{x, y\}^{\perp} A & =(s+1) j-s \cdot\left(x^{\perp}+y^{\perp}\right)+(s-1) \cdot\{x, y\}^{\perp}+s^{2} \cdot\{x, y\}, \\
\{x, y\} A & =x^{\perp}+y^{\perp}-\{x, y\} .
\end{aligned}
$$

So

$$
\begin{aligned}
\mathcal{T} A & =\{x, y\}^{\perp} A+s \cdot(x A)+s \cdot(y A) \\
& =(s+1) j+(s-1)\{x, y\}^{\perp}+\left(s^{2}-s\right)\{x, y\} \\
& =(s+1) j+(s-1) \mathcal{T}
\end{aligned}
$$

It then follows that $v$ is an eigenvector of the adjacency matrix $A$ with eigenvalue $s-1$.

Theorem 5.2. Let $\mathcal{H}$ be a set of points of $\mathcal{Q}$ giving rise to a hemisystem of the dual of $\mathcal{Q}$. Then the geometry $\Gamma$ obtained by restricting the point-set of $\mathcal{Q}$ to $\mathcal{H}$ is a partial quadrangle.

Proof. Clearly every two points of $\Gamma$ share at most one line and there are no triangles in $\Gamma$, so it suffices to prove the " $\mu$ condition". Let $x, y$ be two arbitrary noncollinear points in $\mathcal{H}$ and let $\mu$ be the number of points of $\mathcal{H}$ which are collinear to both $x$ and $y$. Note that $x^{\perp} \cap y^{\perp}$ consists of $s^{2}+1$ points in $\mathcal{Q}$. We simply use the above lemma to show that $\mu$ is independent of the choice of $x$ and $y$. Consider the multiset $\mathcal{T}$ of points consisting of $\{x, y\}^{\perp}$ and $s$ copies of $x$ and $y$. So in other words, this multiset can be expressed by $\mathcal{T}=\{x, y\}^{\perp}+s(\{x\}+\{y\})$. Simply note now that

$$
\frac{|\mathcal{T}||\mathcal{H}|}{(s+1)\left(s^{3}+1\right)}=\mathcal{T} \bullet \mathcal{H}=\{x, y\}^{\perp} \bullet \mathcal{H}+s(\{x\} \bullet \mathcal{H})+s(\{y\} \bullet \mathcal{H})=\mu+2 s
$$

and hence $\mu=\frac{1}{2}\left(\left(s^{2}+1\right)+2 s\right)-2 s=(s-1)^{2} / 2$. Therefore $\Gamma$ is a partial quadrangle. 
J. Bamberg, M. Giudici and G. F. Royle

School of Mathematics and Statistics,

The University of Western Australia,

35 Stirling Highway, Crawley, W.A. 6009

Australia

bamberg@maths.uwa.edu.au

giudici@maths.uwa.edu.au

gordon@maths.uwa.edu.au

\section{References}

[1] L. Bader, N. Durante, M. Law, G. Lunardon, and T. Penttila. Symmetries of BLT-sets. In Proceedings of the Conference on Finite Geometries (Oberwolfach, 2001), volume 29, pages 41-50, 2003.

[2] L. Bader, G. Lunardon, and J. A. Thas. Derivation of flocks of quadratic cones. Forum Math., 2(2):163-174, 1990.

[3] J. Bamberg, F. De Clerck, and N. Durante. A hemisystem of a nonclassical generalised quadrangle. Des. Codes Cryptogr., 51(2):157-165, 2009.

[4] J. Bamberg, M. Giudici, and G. F. Royle. Hemisystems of small flock quadrangles. in preparation.

[5] J. Bamberg, S. Kelly, M. Law, and T. Penttila. Tight sets and $m$-ovoids of finite polar spaces. J. Combin. Theory Ser. A, 114(7):1293-1314, 2007.

[6] J. Bamberg, T. Penttila, and C. Schneider. Elation generalized quadrangles for which the number of lines on a point is the successor of a prime. J. Aust. Math. Soc., 85(3):289-303, 2008.

[7] A. Betten. http://www.math.colostate.edu/ betten/blt.html

[8] R. C. Bose and S. S. Shrikhande. Geometric and pseudo-geometric graphs $\left(q^{2}+1, q+1,1\right)$. J. Geometry, 2:75-94, 1972.

[9] P. J. Cameron. Partial quadrangles. Quart. J. Math. Oxford Ser. (2), 26:61-73, 1975.

[10] P. J. Cameron, P. Delsarte, and J.-M. Goethals. Hemisystems, orthogonal configurations, and dissipative conference matrices. Philips J. Res., 34(3-4):147-162, 1979.

[11] A. Cossidente and T. Penttila. A new hemisystem of $H(3,49)$. to appear in Ars Combinatoria.

[12] A. Cossidente and T. Penttila. Hemisystems on the Hermitian surface. J. London Math. Soc. (2), 72(3):731-741, 2005.

[13] J. W. P. Hirschfeld. Finite projective spaces of three dimensions. Oxford Mathematical Monographs. The Clarendon Press Oxford University Press, New York, 1985. Oxford Science Publications.

[14] J. W. P. Hirschfeld and J. A. Thas. General Galois geometries. Oxford Mathematical Monographs. The Clarendon Press Oxford University Press, New York, 1991. Oxford Science Publications.

[15] N. Knarr. A geometric construction of generalized quadrangles from polar spaces of rank three. Results Math., 21(34):332-344, 1992.

[16] M. Law. Flocks, generalised quadrangles and translation planes from BLT-sets. PhD thesis, The University of Western Australia, 2003.

[17] M. Law and T. Penttila. Classification of flocks of the quadratic cone over fields of order at most 29. Adv. Geom. (suppl.):S232-S244, 2003. Special issue dedicated to Adriano Barlotti.

[18] M. Muzychuk and E. R. van Dam. Cometric $Q$-antipodal association schemes - uniformity and linked systems. preprint.

[19] S. E. Payne. The fundamental theorem of q-clan geometry. Des. Codes Cryptogr., 8(1-2):181-202, 1996. Special issue dedicated to Hanfried Lenz.

[20] S. E. Payne and L. A. Rogers. Local group actions on generalized quadrangles. Simon Stevin, 64(3-4):249-284, 1990.

[21] S. E. Payne and J. A. Thas. Finite generalized quadrangles, volume 110 of Research Notes in Mathematics. Pitman (Advanced Publishing Program), Boston, MA, 1984.

[22] B. Segre. Forme e geometrie hermitiane, con particolare riguardo al caso finito. Ann. Mat. Pura Appl. (4), 70:1-201, 1965.

[23] J. A. Thas. Generalized quadrangles and flocks of cones. European J. Combin., 8(4):441-452, 1987.

[24] J. A. Thas. Projective geometry over a finite field. In Handbook of incidence geometry, pages 295-347. North-Holland, Amsterdam, 1995.

[25] J. A. Thas. Finite geometries: classical problems and recent developments. Rend. Mat. Appl. (7), 27(1):49-60, 2007. 